\title{
Topiramate in the Treatment of Epilepsy - A Review
}

\section{a report by \\ Renzo Guerrini}

Professor of Paediatric Neurology and Head of Neurology Unit, Pediatric Hospital A Meyer and

University of Florence

DOI:10.17925/ENR.2006.00.02.40

\section{Introduction}

Since 1990 around seven new anti-epileptic drugs (AEDs) have joined the marketplace. Among them, topiramate (TPM), a sulphamate-substituted monosaccharide, which was originally synthesised as an oral hypoglycaemic, has proven to be remarkably effective through a wide series of trials.

Mechanism of Action of

Topiramate

Although the exact mechanism of action of TPM remains unknown, multiple mechanisms have been hypothesised based on in vitro studies, including voltage-sensitive sodium channel blockade, $\mathrm{GABA}_{\mathrm{A}}$-mediated chloride current increment, glutamate-mediated neurotransmission inhibition, increase of potassium conductance, carbonic anhydrase isoenzyme inhibition, and interaction with protein kinase phosphorylation sites. The proposed mechanism of action for TPM has been extensively reviewed. ${ }^{1}$

In comparison to other AEDs, TPM has exhibited a modulatory effect on potassium conductance. TPM-induced hyperpolarisation and decrease in input resistance is due to an increase in potassium conductance. $^{2}$

Drugs that potentiate potassium-channels, such as TPM, would be expected to have an anti-epileptic effect by decreasing the excitability of neurons. Lossof-function potassium channel mutations are responsible for benign neonatal convulsions. ${ }^{3}$

\section{Interactions with Other Drugs}

The inhibitory action that TPM exerts on the CYP2C19 isoenzyme may lead to potential interactions with phenytoin and diazepam but known interactions with other drugs are modest and rarely of clinical significance. ${ }^{1}$ An interaction between TPM and oral contraceptives may require a moderate increase in the contraceptive dosage to maintain its effects. ${ }^{1}$

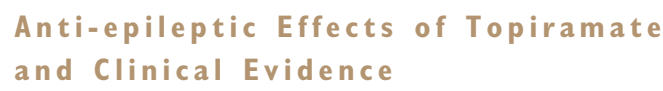

In Europe, TPM is indicated as monotherapy in adults and children aged six years and above with newly diagnosed epilepsy who have generalised tonic-clonic seizures or partial (focal) seizures with or without secondarily generalised seizures.

It is also indicated as adjunctive therapy for epilepsy in children over two years of age and adults with either partial onset or generalised tonic-clonic seizures. Additional indications include the add-on treatment of Lennox-Gastaut syndrome in children and adults.

Topiramate as Monotherapy

TPM as monotherapy in patients with either with drug-resistant or newly diagnosed epilepsies has been studied in three different trials. In a cohort of 48 adults ( $\geq 14$ years of age) with drug-resistant partial epilepsy, TPM was titrated to $1,000 \mathrm{mg} /$ day or to the highest tolerated dose $(n=24)$ or to $100 \mathrm{mg} /$ day $(\mathrm{n}=24)$ over five weeks in a doubleblind fashion. ${ }^{4}$

Concomitant AEDs were tapered off over the same period, whereas maintenance lasted 11 weeks. Time to exit due to seizure deterioration was significantly longer for patients in the high dose group ( $\mathrm{p}=$ 0.002). High doses were associated with better seizure control (46\% versus $13 \%$ of patients with $\geq 50 \%$ seizure reduction) and with higher rates of patients being seizure-free.

A multicentre study analysed the efficacy of TPM as monotherapy in recently ( $\leq 3$ years) diagnosed partial epilepsy. ${ }^{5}$ A total of 252 patients (6-85 years of age) were randomised either to low doses $(25 \mathrm{mg}$ /day if weight $\geq 50 \mathrm{~kg}$, or $50 \mathrm{mg} /$ day, if weight $\leq 50 \mathrm{~kg}$ ) or to high doses $(200 \mathrm{mg} /$ day if weight $\leq 50 \mathrm{~kg}$, or $500 \mathrm{mg} /$ day if weight $\geq 50 \mathrm{~kg}$ ). Time-to-exit due to the recurrence of a second seizure favoured the high doses (422 versus 293 days) without reaching a statistically significant difference. 
However, when time to first seizure (317 versus 108 days) was added as a covariate, the differences between groups reached statistical significance $(\mathrm{p}=$ $0.01)$ as it did for the number of seizure-free patients during the six-month study $(54 \%$ versus $39 \% ; \mathrm{p}=0.02)$.

A multinational, double-blind study in newly $(\leq 3$ months) diagnosed partial epilepsies involved 613 patients ( 6 to $>65$ years of age) from 115 centres. $^{6}$ TPM 100 or $200 \mathrm{mg} /$ day was compared with either carbamazepine (CBZ) $600 \mathrm{mg} /$ day or valproate (VPA) $1,250 \mathrm{mg} /$ day. Patients were allocated to either CBZ or VPA, according to their clinical features, followed by randomisation to receive either the designated comparator drug or TPM 100 or $200 \mathrm{mg} /$ day in double-blind fashion.

Efficacy parameters, represented by time to exit, time to first seizure, and proportion of seizurefree patients during the six-month period of the study, did not differ between groups, showing that all used medications possessed equivalent efficacy. However, TPM 100mg/day was associated with fewer side effects. Further analysis of the paediatric subpop-ulation of the study (6-16 years of age) produced similar results when analysed in more detail. ${ }^{7}$
$5 \%$ of patients treated with TPM and in none of those on placebo.

A placebo-controlled trial of adults with drug-resistant partial epilepsy supported the clinical efficacy of lower doses of TPM seen in the earlier trials. ${ }^{9}$

In the study, 263 adults (18-67 years of age) receiving carbamazepine CBZ were randomised to either placebo $(\mathrm{n}=92)$ or TPM $200 \mathrm{mg} /$ day $(\mathrm{n}=$ 171). The study design was similar to the previous placebo-controlled trials, consisting of a four- to eight-week escalation period and a 12-week maintenance period. The TPM arm reported a median seizure frequency reduction of $44 \%$ versus $20 \%$ in the placebo arm $(\mathrm{p} \leq 0.001)$.

TPM has also been studied in children (2-16 years) with focal epilepsy as adjunctive therapy in a randomised placebo-controlled study. ${ }^{10}$ Thirty-nine percent of patients treated with TPM $(\mathrm{n}=41)$ had a seizure reduction of $>50 \%$ compared with only $20 \%$ on placebo $(n=45)$. TPM had different target doses according to body weight $(125 \mathrm{mg} /$ day for $16-24.9 \mathrm{~kg}$, $175 \mathrm{mg} /$ day for $25-33.9 \mathrm{~kg}, 225 \mathrm{mg} /$ day for $34-42.9 \mathrm{~kg}$ and $400 \mathrm{mg} /$ day for $\geq 43 \mathrm{~kg}$ ). The results were of borderline statistical significance.

\section{TPM was considered to be as effective as standard AEDs} in the treatment of newly diagnosed partial epilepsy in adults and children.

Topiramate in Focal

Epilepsies

The efficacy of TPM as an add-on AED in refractory partial epilepsy in adults has been studied in eight multicentre, randomised, placebocontrolled trials. ${ }^{1}$ In the different trials, either a single dose (200, 400, 600, 800 or $1,000 \mathrm{mg} /$ day) or a range of doses (300-600 or 600-1,000mg/day) were used. Because the eight studies all had a similar design: a titration period of 3-11 weeks followed by $8-12$ weeks of maintenance, a pooled data analysis was possible. ${ }^{8}$ Of the 527 adults with focal, drug-resistant epilepsy treated with TPM, $43 \%$ of the patients experienced a reduction in seizure frequency of $\geq 50 \%$, compared with only $12 \%$ of 216 patients on placebo $(p<0.001)$. Seizure freedom was reported in
However, 5\% of TPM-treated children were seizurefree versus none of those on placebo. An open-label extension of the study showed that $64 \%$ of the children treated for six months had a seizure frequency reduction of $\geq 50 \%$, and $14 \%$ were seizure-free. ${ }^{11}$

Recently published evidence-based guidelines reviewed efficacy and tolerability of new AEDs in the treatment of new onset and refractory epilepsy.

According to classes of evidence, TPM was considered to be as effective as standard AEDs in the treatment of newly diagnosed partial epilepsy in adults and children. Class I evidence was also obtained for TPM use in refractory focal and generalised epilepsy in adults and children, as monotherapy in refractory focal epilepsy in adults, and in Lennox-Gastaut syndrome. ${ }^{12,13}$ 
Topiramate in Generalised Epilepsies

The efficacy of TPM as adjunctive therapy has been studied in 80 patients (3-59 years of age), including 21 children (age 4-16 years) with primary generalised tonic-clonic seizures, with $(67.5 \%)$ or without $(32.5 \%)$ other generalised seizures. ${ }^{14}$

Patients in the trial had an idiopathic generalised epilepsy that was resistant to medications. Patients with Lennox-Gastaut syndrome were excluded from the study.

The study design included eight-week titration and 12-week maintenance phases. Overall, 39 patients were allocated to TPM (adjusted to patient's weight) and 41 to placebo. Median percentage reduction in generalised tonic-clonic seizures was $56.7 \%$ on TPM and $9 \%$ on placebo ( $\mathrm{p}<0.05)$. Considering all generalised seizures, difference between reductions with TPM and placebo was even greater $(p<0.005)$. Response rate $(R R)$ was statistically higher in patients receiving TPM than in those on placebo. common side effect observed in clinical trials. Controlled clinical trials on TPM showed that discontinuation rate related to adverse events ranged from $8-26 \%$ in the TPM arm versus $0-7 \%$ in the placebo arm. ${ }^{13}$

Hypohydrosis associated to TPM administration had been reported in children and adults, although children seem to be more prone to develop this side effect. ${ }^{1}$ Renal calculi have been observed in about $1.5 \%$ of treated patients (82) and have been estimated to be 2-4 times higher than expected in the general population. ${ }^{1}$

TPM treatment has been associated to ocular side effects such as acute glaucoma, acute myopia, suprachoroidal effusion, periorbital oedema, and scleritis in isolated case reports, whose actual frequency remains unclear. ${ }^{1}$ These ocular manifestations are reversible upon drug withdrawal.

Data about the use of TPM in pregnancy have only been collected from add-on use and are therefore inadequate to assess whether any teratogenic potential is present in monotherapy

\section{In clinical trials TPM has been shown to be a relatively well-tolerated and safe AED.}

TPM efficacy as add-on therapy was studied in patients with Lennox-Gastaut syndrome. Patients were assigned to an 11-week, double-blind treatment phase with either TPM $(n=48)$ reaching a maximum dose of $6 \mathrm{mg} / \mathrm{kg} /$ day or placebo $(\mathrm{n}=50) .{ }^{15}$

In the study, $33 \%$ of patients responded to TPM, and $8 \%$ to placebo. Considering seizures causing drop attacks as the most disabling seizure type, TPMtreated patients had a mean reduction of $15 \%$, whereas placebo-treated patients had a $5 \%$ increase.

\section{Safety of Topiramate}

In clinical trials TPM has been shown to be a relatively well-tolerated and safe AED, considering its lack of significant effects on cardiovascular function, bone density, bone marrow cells and thyroid function. The most common side effects responsible for discontinuation of treatment relate to cognitive functions. Weight loss is also a and comparable of other AEDs.

Experimental data suggest that TPM can be teratogenic in mice, rats, and rabbits, producing craniofacial defects, limb and vertebral malformations. ${ }^{16}$

CNS-related Side Effects

CNS-related side effects represented the most common adverse reactions in controlled and openlabel studies. ${ }^{13}$ They consisted mainly of somnolence, fatigue, headache, psychomotor slowing, confusion, difficulty with memory, impaired concentration, attention, speech and language problems. CNS-related side effects were reported by $\leq 40 \%$ of patients who were specifically investigated for this aspect. ${ }^{17}$ Profound language regression has been reported as a reversible side effect of TPM in neurologically impaired children (113). ${ }^{18}$ 
A recent study evaluated the cognitive side effects of tiagabine (TGB) and topiramate. ${ }^{19}$ The study, a comparative open randomized trial with TPM and TGB as add-on therapy, analysed the influence of treatment on cognition, mood, and health-related quality of life (HRQOL).

Forty-one patients with refractory epilepsy were randomly assigned to either TPM or TGB. Intelligence, attention, working memory, episodic memory, and language were assessed at baseline, after titration and during maintenance. Self-report questionnaires were used to assess mood and HRQOL.

From baseline to after the titration paired sampled tests revealed significant deterioration in verbal fluency, language comprehension, working memory and visual block tapping under TPM. These functions remained stable in the maintenance phase.

Self-report measures initially indicated concerns about AED side effects in both groups and
TPM. The mean body weight decrease is $2-7 \%$, occurring early in the course of treatment and reaching its maximum by $15-18$ months. ${ }^{8}$ The reasons behind this side effect is not fully understood. It has been hypothesised that it may include reduction of appetite and inhibition of fat deposition as observed in animal models. ${ }^{21}$

Topiramate Dosing

Data from a large international study with a naturalistic design suggest that TPM should be slowly titrated and used at low-medium dosage with 'flexible' dose adjustments in order to enhance tolerability and increase retention rates. ${ }^{20}$

Good efficacy with an excellent tolerability and safety profile are obtained at doses up to $200 \mathrm{mg}$ /day in adults and to around $3.5 \mathrm{mg} / \mathrm{kg} /$ day in children.

Adults should receive an initial treatment with $25 \mathrm{mg} /$ day in the evening, followed by weekly increments of $25 \mathrm{mg}$ /day during four weeks until reaching the target dose of $100 \mathrm{mg} /$ day, given twice daily as morning and evening doses.

\section{Data from continuing trials and greater clinical experience will help to clarify the specific categories of patients that are most likely to benefit from initial monotherapy with TPM.}

concerns about worse cognitive functioning and depression under TPM. In the maintenance phase patients on TPM demonstrated improvement on all QOLIE scales on a descriptive level.

The authors concluded that TPM intake appeared to be associated with persistent negative cognitive side effects on frontal lobe-associated functions.

However, in the TPM group initially negatively affected HRQOL returned to baseline in the long run on a descriptive level. A relatively common neurological side effect observed in $\leq 60 \%$ of treated patients is paresthesia, which is usually mild and can spontaneously disappear. ${ }^{4,20}$ This side effect might be related to the carbonic anhydrase inhibitory activity associated to TPM.

Weight Loss with Topiramate

Weight loss is a frequently reported side effect with
In children, TPM is better started at $0.5 \mathrm{mg} / \mathrm{kg} / \mathrm{day}$ in the evening, and escalated with $0.5 \mathrm{mg} / \mathrm{kg} /$ day during six weeks to the target dose of $3 \mathrm{mg} / \mathrm{kg} /$ day.

However, patients with refractory epilepsy may benefit from further up-titration until the optimal balance between seizure control and tolerability is reached.

\section{Sum m a ry}

TPM, an AED with multiple putative mechanisms of action, has proven to be remarkably effective through a wide series of trials in several types of epilepsy.

Slow titration of TPM to a low-medium dosage will enhance tolerability. Good efficacy with an excellent tolerability and safety profile are obtained at doses $(200 \mathrm{mg} /$ day in adults and $\sim 3.5 \mathrm{mg} / \mathrm{kg} /$ day in children. Furthermore, long- 
term follow-up studies indicate that the response to TPM is maintained.

Known interactions with other drugs are modest and rarely of clinical significance, however, its inhibitory action on the CYP2C19 isoenzyme may lead to potential interactions with PHT and diazepam. Thus, TPM offers a valid therapeutic option in a wide spectrum of epileptic disorders.

Data from continuing trials and greater clinical experience will help to clarify the specific categories of patients that are most likely to benefit from initial monotherapy with TPM.

References

1. Guerrini R, Parmeggiani L, "Topiramate and its clinical applications in epilepsy", Expert Opin Pharmacother (2006); 7: pp. 811-823.

2. Herrero AI, Del Olmoa N, Gonzalez-Escaladab JR, Solis JM, "Two new actions of topiramate: inhibition of depolarizing GABAA-mediated responses and activation of a potassium conductance", Neuropharmacology (2002);42: $p p$. 210-220.

3. Guerrini R, Casari G, Marini C, "The genetic and molecular basis of epilepsy", Trends Mol Med (2003);9: pp. 300-306.

4. Sachdeo RC, Reife RA, Lim P, Pledger G, "Topiramate monotherapy for partial onset seizures", Epilepsia (1997);38: pp. 294-300.

5. Gilliam FG, Veloso F, Bomhof MA, et al., "A dose-comparison trial of topiramate as monotherapy in recently diagnosed partial epilepsy”, Neurology (2003);60: pp. 196-202.

6. Privitera MD, Brodie MJ, Mattson RH, et al., "Topiramate, carbamazepine and valproate monotherapy: double-blind comparison in newly diagnosed epilepsy”, Acta Neurol Scand (2003);107: pp. 165-175.

7. Wheless JW, Neto $W$, Wang S; EPMN-105 Study Group, "Topiramate, carbamazepine, and valproate monotherapy: double-blind comparison in children with newly diagnosed epilepsy", J Child Neurol (2004);19(2): pp. 135-141.

8. Reife R, Pledger G, Wu SC, "Topiramate as add-on therapy: pooled analysis of randomized controlled trials in adults", Epilepsia (2000);41 Suppl 1: pp. S66-S71.

9. Guberman A, Neto W, Gassmann-Mayer C; EPAJ-119 Study Group, "Low-dose topiramate in adults with treatmentresistant partial-onset seizures”, Acta Neurol Scand (2002);106: pp. 183-189.

10. Elterman RD, Glauser TA, Wyllie E, et al., "A double-blind, randomized trial of topiramate as adjunctive therapy for partial-onset seizures in children. Topiramate YP Study Group”, Neurology (1999);52: pp. 1338-1344.

11. Ritter F, Glauser TA, Elterman RD, Wyllie E, "Effectiveness, tolerability, and safety of topiramate in children with partial-onset seizures. Topiramate YP Study Group”, Epilepsia (2000);41 Suppl 1: pp. S82-S85.

12. French JA, Kanner AM, Bautista J, et al., "Efficacy and tolerability of the new antiepileptic drugs I: treatment of new onset epilepsy: report of the Therapentics and Technology Assessment Subcommittee and Quality Standards Subcommittee of the American Academy of Neurology and the American Epilepsy Society", Neurology (2004);62: pp. 1252-1260.

13. French JA, Kanner AM, Bautista J, et al., "Efficacy and tolerability of the new antiepileptic drugs II: treatment of refractory epilepsy: report of the Therapeutics and Technology Assessment Subcommittee and Quality Standards Subcommittee of the American Academy of Neurology and the American Epilepsy Society", Neurology (2004);62: pp. 1261-1273.

14. Biton V, Montouris GD, Ritter F, et al., "A randomized, placebo-controlled study of topiramate in primary generalized tonic-clonic seizures. Topiramate YTC Study Group”, Neurology (1999);52: pp. 1330-1337.

15. Sachdeo RC, Glauser TA, Ritter F, et al., " $A$ double-blind, randomized trial of topiramate in Lennox-Gastaut syndrome. Topiramate YL Study Group”, Neurology (1999);52: pp. 1882-1887.

16. Privitera MD, Twyman RE, "Topiramate. Clinical efficacy and use in epilepsy", Levy RH, Mattson RH, Meldrum BS, Perucca E, (eds.) Antiepileptic Drugs, Fifth Edition (2002), Philadelphia, Lippincott Williams \& Wilkins: pp. $740-752$.

17. Meador KJ, Loring DW, Hulihan JF, et al., "Differential cognitive and behavioral effects of topiramate and valproate", Neurology (2003)13;60(9): pp. 1483-1488.

18. Gross-Tsur V, Shalev RS, "Reversible language regression as an adverse effect of topiramate treatment in children", Neurology (2004);62(2): pp. 299-300.

19. Fritz N, Glogau S, Hoffmann J, et al., "Efficacy and cognitive side effects of tiagabine and topiramate in patients with epilepsy”, Epilepsy Behav (2005);6: pp. 373-381.

20. Guerrini R, Carpay J, Groselj J, et al., "Topiramate monotherapy as broad-spectrum antiepileptic drug in a naturalistic clinical setting”, Seizure (2005);14(6):371-380.

21. York DA, Singer L, Thomas S, Bray GA, "Effect of topiramate on body weight and body composition of Osborne-Mendel rats fed a high-fat diet: alterations in hormones, neuropeptide, and uncoupling-protein mRNAs", Nutrition (2000);16: pp. 967-975. 


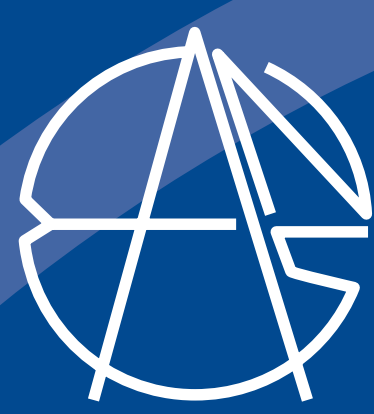

EUROPEAN ASSOCIATION OF NEUROSURGICAL SOCIETIES

“THE EANS - A PARTNERSHIP FOR NEUROSURGERY”

EUROPEAN NATIONAL SOCIETY MEMBERSHIP

$\Leftrightarrow$ INDIVIDUAL MEMBERSHIP

MEETINGS AND CONFERENCES

$\Rightarrow$ TRAINING

The EANS Winter Meeting

Antalya, February 2007

The EANS European Training Course in Neurosurgery

Antalya, February 2007

Lisbon, June 2007

Please consult www.eans.org for further venues

\section{The EANS Research Course}

Lisbon, June 2007

The European Journal of Neurosurgery: "Acta Neurochirurgica"

For all information please contact Stephanie Garfield-Birkbeck

at eans_secretariat@philsparrow.co.uk

www.eans.org 